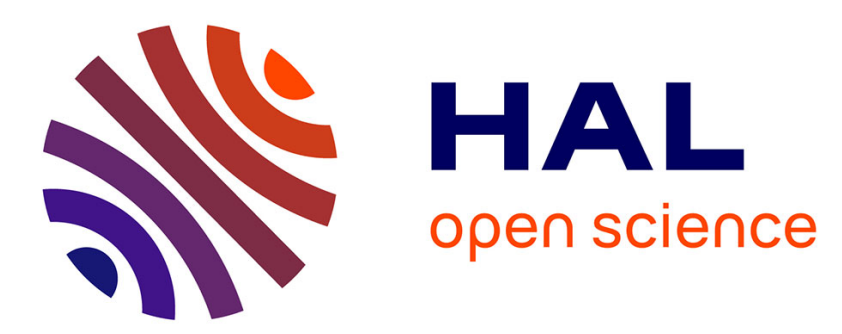

\title{
From neglected to dissected: How technological advances are leading the way to the study of Coxiella burnetii pathogenesis \\ Melanie Burette, Matteo Bonazzi
}

\section{- To cite this version:}

Melanie Burette, Matteo Bonazzi. From neglected to dissected: How technological advances are leading the way to the study of Coxiella burnetii pathogenesis. Cellular Microbiology, 2020, Celebrating the Career and Legacy of Professor Pascale Cossart, 22 (4), pp.e13180. 10.1111/cmi.13180 . hal02993239

\section{HAL Id: hal-02993239 \\ https://hal.science/hal-02993239}

Submitted on 9 Nov 2020

HAL is a multi-disciplinary open access archive for the deposit and dissemination of scientific research documents, whether they are published or not. The documents may come from teaching and research institutions in France or abroad, or from public or private research centers.
L'archive ouverte pluridisciplinaire HAL, est destinée au dépôt et à la diffusion de documents scientifiques de niveau recherche, publiés ou non, émanant des établissements d'enseignement et de recherche français ou étrangers, des laboratoires publics ou privés. 
From neglected to dissected: how technological advances are leading the way to the study of Coxiella burnetii pathogenesis

Melanie Burette ${ }^{1} \&$ Matteo Bonazzi ${ }^{1}$

1. IRIM, UMR 9004 CNRS, Université de Montpellier, Montpellier, France.

For correspondence: matteo.bonazzi@irim.cnrs.fr 


\section{Abstract}

Coxiella burnetii is an obligate intracellular bacterial pathogen responsible for severe worldwide outbreaks of the zoonosis $Q$ fever. The remarkable resistance to environmental stress, extremely low infectious dose and ease of dissemination, contributed to the classification of $C$. burnetii as a class B biothreat. Unique among intracellular pathogens, $C$. burnetii escapes immune surveillance and replicates within large autophagolysosome-like compartments called Coxiella-containing vacuoles (CCVs). The biogenesis of these compartments depends on the subversion of several host signaling pathways. For years, the obligate intracellular nature of $C$. burnetii imposed significant experimental obstacles to the study of its pathogenic traits. With the development of an axenic culture medium in 2009, C. burnetii became genetically tractable, thus allowing the implementation of mutagenesis tools and screening approaches to identify its virulence determinants and investigate its complex interaction with host cells. Here, we review the key advances that have contributed to our knowledge of $C$. burnetii pathogenesis, leading to the rise of this once-neglected pathogen to an exceptional organism to study the intravacuolar lifestyle. 


\section{Introducing Coxiella burnetii}

Coxiella burnetii is a Gram negative obligate intracellular pathogen, and the causative agent of $Q$ fever, a worldwide zoonotic disease (Eldin et al., 2017). Animal coxiellosis is mostly associated with abortion, stillbirths, and weak offspring (Fig. 1). Desiccation of placental materials, excretions of birthing fluids, urine, feces and milk from infected animals contribute to shed C. burnetii into the environment (Eldin et al., 2017). Transmission to humans results from exposure to contaminated aerosols and dust (Fig. 1). In humans, C. burnetii infections are often asymptomatic and self-limiting, however, $40 \%$ of individuals infected with $C$. burnetii develop an acute disease which is associated with a flu-like syndrome, pneumonia, hepatitis and chronic fatigue (Eldin et al., 2017) (Fig. 1). Acute disease may convert into a chronic illness with severe complications, including endocarditis (Eldin et al., 2017). An important risk factor for $Q$ fever outbreaks is the multiple zoonotic reservoirs of $C$. burnetii, which include domestic livestock and wild animals (mammals, reptiles, birds and ticks) (Eldin et al., 2017). C. burnetii is extremely infectious, with 1-to-10 bacteria being sufficient to cause disease (Brooke et al., 2013). The low infectious dose coupled to remarkable environmental stability contribute to the significant spreading of $C$. burnetii infections well away from the outbreak source and led to the classification of $C$. burnetii as a category B biothreat (Madariaga et al., 2003).

C. burnetii is a stealth pathogen that actively escapes innate immune recognition by inhibiting the NF-kB pathway (Mahapatra et al., 2016) and inflammasome activation (Cunha et al., 2015). Infected cells are also protected from apoptosis, thereby preserving the bacterial replicative niche over long periods (Lührmann et al., 2017). C. burnetii enters macrophages by phagocytosis through the interaction with $\alpha \mathrm{V} \beta 3$ integrins (Capo et al., 1999). In contrast, in non-phagocytic cells, 
internalization is facilitated by the invasin OmpA (Outer membrane protein A) (Martinez et al., 2014) (Fig. 1). Following internalization, bacteria reside within early endosomal compartments, also called early CCVs, that passively mature along the endocytic pathway by successive fusion events with early and late endosomes and lysosomes (Fig. 1). Maturation is accompanied by the acidification of the CCV lumen (Heinzen et al., 1996), which is required to activate bacterial metabolism (Hackstadt and Williams, 1981) and the translocation of bacterial effector proteins by a Dot/lcm Type $4 \mathrm{~b}$ Secretion System (T4SS) (Newton et al., 2013). Thus, by 48 hours post-infection, cells display a single, large, mature $\mathrm{CCV}$, where markers of multiple membrane compartments co-exist (Dragan and Voth, 2019), which is indicative of the capacity of C. burnetii to hijack multiple host membrane trafficking pathways (Fig. 1). Importantly, the biogenesis of these remarkable compartments is a two-step process requiring both cellular and bacterial factors. This review will focus on the recent technological advances that fostered remarkable progress in our understanding of the complex host/pathogen interplay that controls the generation of the $C$. burnetii replicative niche.

\section{The long and winding road (to genetic manipulation)}

Together with its high infectivity, the obligate intracellular nature of $C$. burnetii has imposed severe constraints on the study of its pathogenesis. An important step towards the development of tools to investigate $C$. burnetii infection was the isolation of a Phase II variant (NMII) of the Nine Mile Phase I strain (NMI), presenting a single deletion of 21 genes involved in lipopolysaccharide biosynthesis and displaying lossof-virulence phenotype in animal models (Moos and Hackstadt, 1987; Hoover et al., 2002). Thus, NMII was allowed for manipulation in biosafety level-2 (BSL-2) environments (Fig. 2D), as opposed to other $C$. burnetii strains that require 
manipulation in biosafety level-3 (BSL-3) confinement. Importantly, loss-of-virulence of NMII has no impact either on intracellular growth kinetics or development of CCVs in cultured cells, making NMII a relevant model to study host-pathogen interactions (Howe et al., 2010).

Nevertheless, bacterial amplification of $C$. burnetii in embryonated eggs and/or cultured cells represented a real obstacle for its genetic manipulation. Indeed, transposon mutagenesis allowed the generation of $C$. burnetii mutants (Beare et al., 2009); however, amplification and isolation of these from infected cells ruled out the possibility of isolating mutations in virulence genes. Thus, until 2011 , fts $Z$ was the only C. burnetii gene characterized using Himar1 transposon mutagenesis (Beare et al., 2009) (Fig. 2F).

Whole-genome sequencing of C. burnetii NMI RSA493 in 2003 allowed the identification of typical features, including a Dot/lcm secretion system, which is highly homologous to that of L. pneumophila (Seshadri et al., 2003) (Fig. 2C). This finding was pivotal to develop bioinformatics-based approaches to predict effector proteinscoding genes. These revealed that similar to L. pneumophila, effector proteins translocated by $C$. burnetii encode a C-terminal secretion signal called the E-block motif and are mostly under the control of a PmrA response regulator (Zusman et al., 2007). Due to the genetic intractability of $C$. burnetii at that time, candidate effector proteins were tested for secretion using L. pneumophila as a surrogate model, exploiting the homologies between the Dot/lcm secretion systems of the two pathogens. This approach allowed the Roy laboratory to validate the translocation of four $C$. burnetii effector proteins encoding ankyrin repeat homology domains (ARHDs) (Pan et al., 2008) (Fig. 2E). Later work from the Samuel laboratory reported the first 
large-scale, bioinformatics-based identification of $C$. burnetii effector proteins and used the L. pneumophila system to validate the secretion of 32 new T4SS substrates (Chen et al., 2010) (Fig. 2H). Moreover, using a shuttle plasmid system for the expression of recombinant proteins in $C$. burnetii, Chen and colleagues also demonstrated that the functionality of the Dot/lcm secretion system (Chen et al., 2010).

One year later, a genetic screen of $C$. burnetii proteins carrying $\mathrm{C}$-terminal secretion signals led to the identification of additional effector proteins (Carey et al., 2011) (Fig. 2l). For this study, wild type (wt) L. pneumophila or the T4SSdefective $\triangle d o t A$ mutant were transformed with a library containing adenylate cyclase enzyme (CyaA)-tagged random fragments of $C$. burnetii genome, leading to the validation of 7 additional $C$. burnetii effectors (Carey et al., 2011). These were further tested for their intracellular localization and function, which indicated a possible implication in the manipulation of host membrane trafficking. Of note, this is the first study to report a replication phenotype associated with a transposon insertion in the C. burnetii Dot/lcm gene icmL (icmL::Tn), thus demonstrating the importance of C. burnetii effector protein translocation for infection (Carey et al., 2011) (Fig. 2I). Today, the development of tailored bioinformatics algorithms for the identification of T4SS effector proteins led to the identification of over 140 candidate $C$. burnetii T4SS effectors (Voth et al., 2009; Chen et al., 2010; Carey et al., 2011; Maturana et al., 2013; Weber et al., 2013). Recent genome comparison of the C. burnetii strains NMII RSA493, Henzerling RSA331, G Q212, K Q154 and Dugway 5J108-111, revealed that many genes encoding candidate effector proteins are either pseudogenized or missing altogether, leaving only 44 out of the 143 identified effector protein-coding genes intact across all strains (Larson et al., 2016). Despite these significant advances, functional 
analysis of $C$. burnetii genes involved in virulence was still limited by the genetic intractability of this pathogen.

A game-changer in the study of $C$. burnetii infections has been the development of a synthetic medium (ACCM for Acidified Citrate Cysteine Medium), followed by the development of ACCM-2, allowing the extracellular culture of this obligate intracellular bacterium (Omsland et al., 2009; Omsland et al., 2011) (Fig. 2G). ACCM-2 development stems from metabolic requirement studies using microarrays, genomic reconstruction of metabolic pathways and metabolite typing (Omsland et al., 2009; Omsland et al., 2011). These studies highlighted that $C$. burnetii requires acid activation buffer ( $\mathrm{pH} 4.75)$, to reproduce the CCV microenvironment (Heinzen et al., 1996) and increase its metabolic potential as well as low oxygen levels $(2.5 \%)$ to facilitate the bacterial micro-aerophilic respiration (Omsland et al., 2009; Omsland et al., 2011). This scientific milestone finally enabled genetic manipulation of $C$. burnetii, leading to a new era in our understanding of its pathogenesis (Fig. 2).

\section{From genes to function: characterization of $C$. burnetii effectors involved in vacuole biogenesis}

With the development of axenic culture, targeted deletion of $C$. burnetii was formally achievable (albeit remaining extremely challenging), and the role of $C$. burnetii effector proteins in infection could be tested directly. The Heinzen laboratory first exploited axenic culture with the generation of a $\operatorname{dot} A$ deletion mutant (Beare et al., 2012) (Fig. 2J) and with the identification and characterization of CvpA (for Coxiella vacuolar protein A). This effector protein localizes at CCVs and reroutes recycling endosomes to this compartment by interacting with the clathrin adaptor protein AP2 (Larson et al., 2013) (Fig. 1). In a follow-up study, the Heinzen laboratory identified four 
additional members of what constitutes today the Cvp sub-class of $C$. burnetii effector proteins (CvpA, CvpB/Cig2, and CvpC-to-E, Fig. 1) (Larson et al., 2015). Accordingly, targeted deletion of $C$. burnetii vacuolar proteins severely affects the biogenesis of CCVs (Larson et al., 2013; Larson et al., 2015).

If targeted deletion of $C$. burnetii genes remains challenging, Himar1-based transposon mutagenesis has been extensively applied to the generation of libraries of C. burnetii mutants. Thus, by 2013 , the Samuel laboratory reported the first study that combined bioinformatics-mediated identification of candidate effector proteins with transposon mutagenesis (Weber et al., 2013) (Fig. 2N). Searching the C. burnetii genome for T4SS features (PmrA consensus sequences, E-block motifs, and homologies with known effectors), led to the identification of 234 genes encoding putative $C$. burnetii effector proteins. $\beta$-lactamase translocation assay in L. pneumophila validated 53 T4SS substrates, most of which were never reported before. Transposon mutagenesis showed that ten effector proteins were involved in the biogenesis of CCVs and bacterial replication (Weber et al., 2013) (Fig. 2N).

The following year, two independent studies reported the large-scale identification of $C$. burnetii virulence determinants based on the generation of two libraries of $C$. burnetii transposon mutants (Martinez et al., 2014; Newton et al., 2014) (Fig. 2O, R). The Bonazzi library consisted of 3000 GFP-tagged mutants, among which over 1000 were sequenced, annotated, and screened using quantitative, multiparametric image analysis to identify bacterial factors involved in host cell invasion, intracellular replication and persistence (Martinez et al., 2014; Martinez et al., 2015). This approach allowed to validate the function of 16 out of the 22 genes constituting the C. burnetii Dot/lcm secretion system, characterize the phenotype associated with transposon insertions in 31 genes encoding effector proteins and 
identify the first $C$. burnetii invasin OmpA (for Outer membrane protein A), which is necessary and sufficient to trigger internalization by non-phagocytic cells (Martinez et al., 2014) (Fig. $1 \& 2 \mathrm{P}$ ). This study was also the first to report the use of the insect model Galleria mellonella to investigate C. burnetii virulence in vivo (Martinez et al., 2014).

The Roy laboratory used a modified-Himar1 expressing mCherry fluorescent protein to generate over 3200 mutants. These were visually screened in HeLa cells to identify genes important in the biogenesis of CCVs and bacterial replication using the lysosomal marker LAMP-1 to visualize the membranes of the $C$. burnetii replicative compartment (Newton et al., 2014) (Fig. 2O). This approach allowed the isolation of mutants characterized by different intracellular phenotypes, including defects in intracellular replication and homotypic fusion of independent CCVs or the appearance of filamentous bacteria. Of note, mutants carrying a transposon insertion in gene encoding CBU1751 (cig57) displayed a severe vacuole biogenesis defect (Newton et al., 2014). The Newton laboratory has further characterized the role of this effector protein in the development of CCVs and intracellular replication, showing that Cig57 subverts clathrin-mediated traffic by interacting with $\mathrm{FCHO} 2$, an accessory protein of clathrin-coated pits (Latomanski et al., 2016) (Fig. 1). More recently, CTLC (clathrin heavy chain) has been observed at CCVs, where it plays an essential role in vacuole expansion (Latomanski and Newton, 2018). Importantly, CTLC recruitment to the CCV is related to autophagy, and conversely, the fusion of autophagosomes with CCVs is dependent on CTLC (Latomanski and Newton, 2018). The Roy visual screen also highlighted a multivacuolar phenotype associated with transposon insertions in the gene CBU0021 (cig2), identical to that previously observed following the knockdown of autophagy-related genes (McDonough et al., 2013), suggesting a functional link 
between a bacterial effector protein and the manipulation of a specific host cell function

(Fig. 20). This hypothesis was further investigated, demonstrating that Cig2 contributes to the recruitment of the autophagy machinery to CCVs (Fig. 1), thus facilitating their homotypic fusion and contributing to an enhanced bacterial virulence in the Galleria mellonella infection model (Kohler et al., 2016).

\section{It takes two to tango: role of host cell pathways in $C$. burnetii vacuole biogenesis}

If on one hand effector proteins translocation is critical for pathogenesis, host cell proteins, lipids, and membrane trafficking pathways also play a significant role in C. burnetii infections. As mentioned above, the maturation of CCVs along the endocytic pathway is a pre-requisite for effector proteins translocation (Newton et al., 2013)(Fig. 2L); thus, Rab GTPases of the endocytic pathway play a key role in C. burnetii intracellular replication (Fig. 1) (Beron et al., 2002; Romano et al., 2007; Campoy et al., 2011). Indeed, silencing of either Rab5 or Rab7 correlates with effector protein translocation defects (Newton et al., 2016) (Fig. 2R).

A comprehensive characterization of the host cell components required for the biogenesis of CCVs was provided for the first time by a genome-wide screen using siRNA targeting eukaryotic genes in C. burnetii-infected HeLa cells (McDonough et al., 2013) (Fig. 2M). Host determinants required for $C$. burnetii infection were identified by the analysis of the number and size of CCVs, revealing the importance of several eukaryotic pathways in critical infection events. As expected, the silencing of genes encoding pH-regulating proteins CLN3 and CLCN5, as well as components of the vacuolar ATPase resulted in a defect in the biogenesis of CCVs (McDonough et al., 2013). Besides, the depletion of the retromer cargo complex VPS26-VPS29-VPS35 leads to defective in bacterial replication, revealing a role for retrograde membrane 
trafficking in C. burnetii infections (McDonough et al., 2013). Interestingly, seminal work from the Colombo laboratory in the past decade demonstrated that the induction of autophagy favors the biogenesis of $\mathrm{CCV}$ s and that $C$. burnetii actively manipulates autophagy during infections (Gutierrez et al., 2005; Romano et al., 2007). However, the precise role of autophagy in CCVs' development remained to be characterized. The genome-wide screening approach of the Roy laboratory revealed that knockdown of autophagy proteins syntaxin-17, ATG5 and ATG12 results in the formation of multiple CCVs of smaller size as compared to control cells (McDonough et al., 2013). It was later demonstrated that this multivacuolar phenotype is the result of the defective homotypic fusion of CCVs (Newton et al., 2014; Martinez et al., 2016).

The Voth and Heinzen laboratories have further explored the complex interplay between $C$. burnetii and autophagy by. The autophagy-associated cargo receptor p62 is actively recruited at CCVs, independently of LC3-interacting domains (Winchell et al., 2018). Interestingly, C. burnetii infections seem to preserve p62 from degradation upon induction of autophagy (Winchell et al., 2018). Accordingly, C. burnetii infections inhibit mTORC1, a master regulator of autophagy, by a non-canonical mechanism that does not result in accelerated autophagy, nor a block of the autophagic flux (Larson et al., 2019). Finally, autophagy is also involved in repairing the membranes of CCVs during expansion, which are subject to transitory damage and loss-of-acidification (Mansilla Pareja et al., 2017) (Fig. 2T).

Besides host cell proteins, lipids also play a significant role in the development of CCVs, which are rich in sterols (Gilk et al., 2010). Indeed, cholesterol homeostasis regulates CCVs biogenesis and intracellular survival of C. burnetii (Gilk et al., 2013; Mulye et al., 2017) (Fig. 2K). Accordingly, C. burnetii actively manipulates cholesterol metabolism via a eukaryotic-like $\Delta 24$ sterol reductase (Gilk et al., 2010). Furthermore, 
an image-based supervised machine learning approach led to the identification of CvpB/Cig2 as the first C. burnetii effector protein to bind phosphoinositides and manipulate their metabolism (Martinez et al., 2016) (Fig. 2S). Indeed, CvpB/Cig2 binds phosphatidylinositol-3-phosphate $(\mathrm{PI}(3) \mathrm{P})$ and phosphatidylserine $(\mathrm{PS})$ and perturbs the activity of the PI3-kinase PIKFYVE. This inhibition results in an enrichment of $\mathrm{PI}(3) \mathrm{P}$ at CCVs, which is essential for their homotypic fusion (Martinez et al., 2016). Importantly, despite the apparent defect in the biogenesis of CCVs, cvpB transposon mutants were unaffected in their capacity of replicating within infected cells. However, $c v p B$ mutants are attenuated in the in vivo models Galleria mellonella (Martinez et al., 2016) and SCID mice (van Schaik et al., 2017), demonstrating that the biogenesis of CCVs can modulate $C$. burnetii virulence, independently of bacterial replication.

\section{To infinity and beyond (conclusions)}

As of today, C. burnetii stands as the sole example of an obligate intracellular bacterial pathogen for which a specific axenic culture medium has been developed. Consequently, research on this dangerous and complex pathogen has bloomed during this decade, with the development of genetic tools and phenotypic screening approaches to better understand the complex interactions established between C. burnetii and its host. The generation of libraries of $C$. burnetii transposon mutants combined with the development of multi-parametric screening approaches have allowed the rapid and unbiased identification of microbial genes involved in specific steps of the infectious process. This has been facilitated by the fact that the majority

of $C$. burnetii transposon mutants isolated to date display a significant phenotype during infection (Martinez et al., 2014), suggesting a milder functional gene redundancy as compared to the closely related pathogen L. pneumophila. Accordingly, 
bioinformatics predicted $\sim 150$ effector proteins compared to the 300 translocated by Legionella.

Nevertheless, understanding how these newly identified virulence determinants manipulate host cell functions remains exceptionally challenging. Probably due to the complex nature of this compartment, a large proportion of the $C$. burnetii effector proteins identified and characterized to date are involved in the biogenesis of the CCV; however, proteins involved in the manipulation of other signaling pathways including apoptosis and inflammation have been identified and partially characterized. With the development of advanced bioinformatics approaches such as unsupervised machine learning, hierarchical clustering and Bayesian network analysis, we can now compare microbial-targeted and host-targeted phenotypic screens, to identify sets of bacterial and eukaryotic genes predicted to be involved in the same biological process during infection.

Despite the giant leaps taken since the development of axenic culture, many technical barriers still exist today. Directed mutagenesis remains challenging, limiting our studies to the mutants available in the transposon libraries hosted in the laboratories that undertook this endeavor. Nevertheless, our knowledge of this onceneglected pathogen is ever increasing. This will allow, in the near future, to address burning questions on $C$. burnetii pathogenesis, including a characterization of the strategies used to evade immune recognition, cell-to-cell spread, and, consequently, dissemination to distant organs (heart and liver) following infection of alveolar macrophages. 


\section{Acknowledgements}

Work in our laboratory is supported by the Agence Nationale de la Recherche (ANR; ANR-14-CE14-0012-01, project AttaQ and ANR- 17-CE15-0021, project QPID) and by the ERA-NET Infect-ERA (ANR-13-IFEC-0003, project EUGENPATH). We apologize to all colleagues whose work was not discussed in this review.

\section{Conflict of interest}

The authors declare no conflict of interest.

\section{References}

Beare, P. A, Larson, C.L., Gilk, S.D., and Heinzen, R. A (2012) Two systems for targeted gene deletion in Coxiella burnetii. Appl Environ Microbiol 78: 4580-9. Beare, P.A., Howe, D., Cockrell, D.C., Omsland, A., Hansen, B., and Heinzen, R.A. (2009) Characterization of a Coxiella burnetii ftsZ mutant generated by Himar1 transposon mutagenesis. J Bacteriol 191: 1369-1381

Beron, W., Gutierrez, M.G., Rabinovitch, M., and Colombo, M.I. (2002) Coxiella burnetii Localizes in a Rab7-Labeled Compartment with Autophagic Characteristics. Infect Immun 70: 5816-5821

Brooke, R.J., Kretzschmar, M.E.E., Mutters, N.T., and Teunis, P.F. (2013) Human dose response relation for airborne exposure to Coxiella burnetii. BMC Infect Dis 13: 488 BMC Infectious Diseases.

Campoy, E.M., Zoppino, F.C.M., and Colombo, M.I. (2011) The Early Secretory Pathway Contributes to the Growth of the Coxiella -Replicative Niche. Infect Immun 79: $402-413$

Capo, C., Lindberg, F.P., Meconi, S., Zaffran, Y., Tardei, G., Brown, E.J., et al. 
(1999) Subversion of monocyte functions by coxiella burnetii: impairment of the cross-talk between alphavbeta3 integrin and CR3. J Immunol 163: 6078-85

Carey, K.L., Newton, H.J., Lührmann, A., and Roy, C.R. (2011) The Coxiella burnetii Dot/Icm System Delivers a Unique Repertoire of Type IV Effectors into Host Cells and Is Required for Intracellular Replication. PLoS Pathog 7: e1002056

Chen, C., Banga, S., Mertens, K., Weber, M.M., Gorbaslieva, I., Tan, Y., et al. (2010) Large-scale identification and translocation of type IV secretion substrates by Coxiella burnetii. Proc Natl Acad Sci 107: 21755-21760.

Cunha, L.D., Ribeiro, J.M., Fernandes, T.D., Massis, L.M., Khoo, C.A., Moffatt, J.H., et al. (2015) Inhibition of inflammasome activation by Coxiella burnetii type IV secretion system effector IcaA. Nat Commun 6: 10205

Dragan, A.L., and Voth, D.E. (2019) Coxiella burnetii: international pathogen of mystery. Microbes Infect 1-11

Eldin, C., Mélenotte, C., Mediannikov, O., Ghigo, E., Million, M., Edouard, S., et al. (2017) From Q Fever to Coxiella burnetii Infection: a Paradigm Change. Clin Microbiol Rev 30: 115-190

Gilk, S.D., Beare, P.A., and Heinzen, R.A. (2010) Coxiella burnetii expresses a functional $\Delta 24$ sterol reductase. J Bacteriol 192: 6154-6159.

Gilk, S.D., Cockrell, D.C., Luterbach, C., Hansen, B., Knodler, L.A., Ibarra, J.A., et al. (2013) Bacterial Colonization of Host Cells in the Absence of Cholesterol. PLoS

Pathog 9.

Gutierrez, M.G., Vázquez, C.L., Munafó, D.B., Zoppino, F.C.M., Berón, W., Rabinovitch, M., and Colombo, M.I. (2005) Autophagy induction favours the generation and maturation of the Coxiella-replicative vacuoles. Cell Microbiol 7: 98193 
Hackstadt, T., and Williams, J.C. (1981) Biochemical stratagem for obligate parasitism of eukaryotic cells by Coxiella burnetii. Proc Natl Acad Sci 78: 3240-3244 Heinzen, R.A., Scidmore, M.A., Rockey, D.D., and Hackstadt, T. (1996) Differential interaction with endocytic and exocytic pathways distinguish parasitophorous vacuoles of Coxiella burnetii and Chlamydia trachomatis. Infect Immun 64: 796-809. Hoover, T.A., Culp, D.W., Vodkin, M.H., Williams, J.C., and Thompson, H.A. (2002) Chromosomal DNA Deletions Explain Phenotypic Characteristics of Two Antigenic Variants, Phase II and RSA 514 (Crazy), of the Coxiella burnetii Nine Mile Strain. Infect Immun 70: 6726-6733

Howe, D., Shannon, J.G., Winfree, S., Dorward, D.W., and Heinzen, R.A. (2010) Coxiella burnetii phase I and II variants replicate with similar kinetics in degradative phagolysosome-like compartments of human macrophages. Infect Immun 78: 34653474 .

Kohler, L.J., Reed, S.R., Sarraf, S.A., Arteaga, D.D., Newton, H.J., and Roy, C.R. (2016) Effector Protein Cig2 Decreases Host Tolerance of Infection by Directing Constitutive Fusion of Autophagosomes with the Coxiella -Containing Vacuole. MBio 7

Larson, C.L., Beare, P.A., Howe, D., and Heinzen, R.A. (2013) Coxiella burnetii effector protein subverts clathrin-mediated vesicular trafficking for pathogen vacuole biogenesis. Proc Natl Acad Sci 110: E4770-E4779

Larson, C.L., Beare, P.A., Voth, D.E., Howe, D., Cockrell, D.C., Bastidas, R.J., et al. (2015) Coxiella burnetii Effector Proteins That Localize to the Parasitophorous Vacuole Membrane Promote Intracellular Replication. Infect Immun 83: 661-670. Larson, C.L., Martinez, E., Beare, P.A., Jeffrey, B., Heinzen, R.A., and Bonazzi, M. (2016) Right on Q: Genetics begin to unravel Coxiella burnetii host cell interactions. 
Future Microbiol 11: 919-939.

Larson, C.L., Sandoz, K.M., Cockrell, D.C., and Heinzen, R.A. (2019) Noncanonical Inhibition of mTORC1 by Coxiella burnetii Promotes Replication within a Phagolysosome-Like Vacuole. 10: 1-16.

Latomanski, E.A., and Newton, H.J. (2018) Interaction between autophagic vesicles and the Coxiella-containing vacuole requires CLTC (clathrin heavy chain). Autophagy 14: $1710-1725$

Latomanski, E.A., Newton, P., Khoo, C.A., and Newton, H.J. (2016) The Effector Cig57 Hijacks FCHO-Mediated Vesicular Trafficking to Facilitate Intracellular Replication of Coxiella burnetii. PLoS Pathog 12: 1-24.

Lührmann, A., Newton, H.J., and Bonazzi, M. (2017) Beginning to understand the role of the Type IV secretion system effector proteins in coxiella burnetii pathogenesis. Curr Top Microbiol Immunol 413: 243-268.

Madariaga, M.G., Rezai, K., Trenholme, G.M., and Weinstein, R.A. (2003) Q fever: a biological weapon in your backyard. Lancet Infect Dis 3: 709-721

Mahapatra, S., Gallaher, B., Smith, S.C., Graham, J.G., Voth, D.E., and Shaw, E.I. (2016) Coxiella burnetii Employs the Dot/Icm Type IV Secretion System to Modulate Host NF-kB/RelA Activation. Front Cell Infect Microbiol 6: 1-13.

Mansilla Pareja, M.E., Bongiovanni, A., Lafont, F., and Colombo, M.I. (2017)

Alterations of the Coxiella burnetii Replicative Vacuole Membrane Integrity and Interplay with the Autophagy Pathway. Front Cell Infect Microbiol 7: 1-17.

Martinez, E., Allombert, J., Cantet, F., Lakhani, A., Yandrapalli, N., Neyret, A., et al. (2016) Coxiella burnetii effector CvpB modulates phosphoinositide metabolism for optimal vacuole development. Proc Natl Acad Sci 113: E3260-E3269 Martinez, E., Cantet, F., and Bonazzi, M. (2015) Generation and multi-phenotypic 
high-content screening of Coxiella burnetii transposon mutants. J Vis Exp 2015 Martinez, E., Cantet, F., Fava, L., Norville, I., and Bonazzi, M. (2014) Identification of OmpA, a Coxiella burnetii Protein Involved in Host Cell Invasion, by Multi-Phenotypic High-Content Screening. PLoS Pathog 10: e1004013

Maturana, P., Graham, J.G., Sharma, U.M., and Voth, D.E. (2013) Refining the plasmid-encoded type IV secretion system substrate repertoire of Coxiella burnetii. $J$ Bacteriol .

McDonough, J.A., Newton, H.J., Klum, S., Swiss, R., Agaisse, H., and Roy, C.R. (2013) Host Pathways Important for Coxiella burnetii Infection Revealed by GenomeWide RNA Interference Screening. MBio 4: 1-13

Moos, A., and Hackstadt, T. (1987) Comparative virulence of intra- and interstrain lipopolysaccharide variants of Coxiella burnetii in the guinea pig model. Infect Immun 55: $1144-1150$.

Mulye, M., Samanta, D., Winfree, S., Heinzen, R.A., and Gilk, S.D. (2017) Elevated cholesterol in the Coxiella burnetii intracellular niche is bacteriolytic. MBio 8.

Newton, H.J., Kohler, L.J., McDonough, J.A., Temoche-Diaz, M., Crabill, E., Hartland, E.L., and Roy, C.R. (2014) A Screen of Coxiella burnetii Mutants Reveals Important Roles for Dot/lcm Effectors and Host Autophagy in Vacuole Biogenesis. PLoS Pathog 10: e1004286

Newton, H.J., McDonough, J.A., and Roy, C.R. (2013) Effector Protein Translocation by the Coxiella burnetii Dot/lcm Type IV Secretion System Requires Endocytic Maturation of the Pathogen-Occupied Vacuole. PLoS One 8: e54566 Newton, P., Latomanski, E.A., and Newton, H.J. (2016) Applying Fluorescence Resonance Energy Transfer (FRET) to Examine Effector Translocation Efficiency by Coxiella burnetii during siRNA Silencing. J Vis Exp 
Omsland, A., Beare, P.A., Hill, J., Cockrell, D.C., Howe, D., Hansen, B., et al. (2011) Isolation from Animal Tissue and Genetic Transformation of Coxiella burnetii Are Facilitated by an Improved Axenic Growth Medium. Appl Environ Microbiol 77: 3720_ 3725

Omsland, A., Cockrell, D.C., Howe, D., Fischer, E.R., Virtaneva, K., Sturdevant, D.E., et al. (2009) Host cell-free growth of the $Q$ fever bacterium Coxiella burnetii. Proc Natl Acad Sci 106: 4430-4434

Pan, X., Lührmann, A., Satoh, A., Laskowski-Arce, M.A., and Roy, C.R. (2008) Ankyrin repeat proteins comprise a diverse family of bacterial type IV effectors. Science (80- ) 320: 1651-1654.

Romano, P.S., Gutierrez, M.G., Berón, W., Rabinovitch, M., and Colombo, M.I. (2007) The autophagic pathway is actively modulated by phase II Coxiella burnetii to efficiently replicate in the host cell. Cell Microbiol 9: 891-909

Schaik, E.J. van, Case, E.D., Martinez, E., Bonazzi, M., and Samuel, J.E. (2017) The SCID mouse model for identifying virulence determinants in Coxiella burnetii. Front Cell Infect Microbiol 7: 1-10.

Seshadri, R., Paulsen, I.T., Eisen, J.A., Read, T.D., Nelson, K.E., Nelson, W.C., et al. (2003) Complete genome sequence of the Q-fever pathogen Coxiella burnetii. Proc Natl Acad Sci 100: 5455-5460

Voth, D.E., Howe, D., Beare, P.A., Vogel, J.P., Unsworth, N., Samuel, J.E., and Heinzen, R.A. (2009) The Coxiella burnetii ankyrin repeat domain-containing protein family is heterogeneous, with C-terminal truncations that influence Dot/lcm-mediated secretion. J Bacteriol 191: 4232-4242.

Weber, M.M., Chen, C., Rowin, K., Mertens, K., Galvan, G., Zhi, H., et al. (2013) Identification of Coxiella burnetii type IV secretion substrates required for intracellular 
replication and Coxiella-containing vacuole formation. J Bacteriol 195: 3914-3924. Winchell, C.G., Dragan, A.L., Brann, K.R., Onyilagha, F.I., Kurten, R.C., and Voth, D.E. (2018) Coxiella burnetii Subverts p62/Sequestosome 1 and Activates Nrf2 Signaling in Human Macrophages . Infect Immun 86.

Zusman, T., Aloni, G., Halperin, E., Kotzer, H., Degtyar, E., Feldman, M., and Segal, G. (2007) The response regulator PmrA is a major regulator of the icm/dot type IV secretion system in Legionella pneumophila and Coxiella burnetii. Mol Microbio/ 63: $1508-1523$ 


\section{Figure legends}

Figure 1. Overview of C. burnetii infections. C. burnetii is an obligate intracellular pathogen which infects wild and farm animals. Bacteria are shed in the environment with birth products and excretions leading to the contamination of hay and dust. Human infection occurs 2-3 weeks after the inhalation of contaminated particles, followed by the internalization of $C$. burnetii by alveolar macrophages. Human $Q$ fever remain asymptomatic in $60 \%$ of infected individuals, whereas $40 \%$ develop an acute, febrile disease, which can turn into a chronic disease with more severe symptoms including endocarditis. C. burnetii invades eukaryotic cell through phagocytosis, which is facilitated by the bacterial invasin OmpA. Early CCVs mature along the endocytic pathway by successive fusion events with early endosomes (EEs), late endosomes (LEs) and lysosomes. Acidification of the CCV activates bacterial metabolism and the translocation of bacterial effector proteins (purple circles and ovals) by the Dot/lcm secretion system. Several effector proteins are collectively beneficial for the biogenesis of the mature CCV where bacterial replication occurs. These include the Cvp family (for Coxiella vacuolar proteins) of effectors that localize at CCV membranes and manipulate host membrane trafficking pathways. CvpA interacts with the clathrin adaptor AP2 at recycling endosomes (REs), re-routing these compartments to the forming CCV. CvpB binds $\mathrm{PI}(3) \mathrm{P}$ at early endosomes and enriches this phospholipid at CCVs. This is required to favor the autophagy-mediated homotypic fusion between CCVs. Although not a Cvp, Cig57 interacts with the clathrin adaptor FCHO2 at clathrincoated pits and re-routes clathrin-mediated membrane traffic to the CCV. 
Figure 2. Milestone discoveries that have contributed to the knowledge of C. burnetii pathogenesis. 


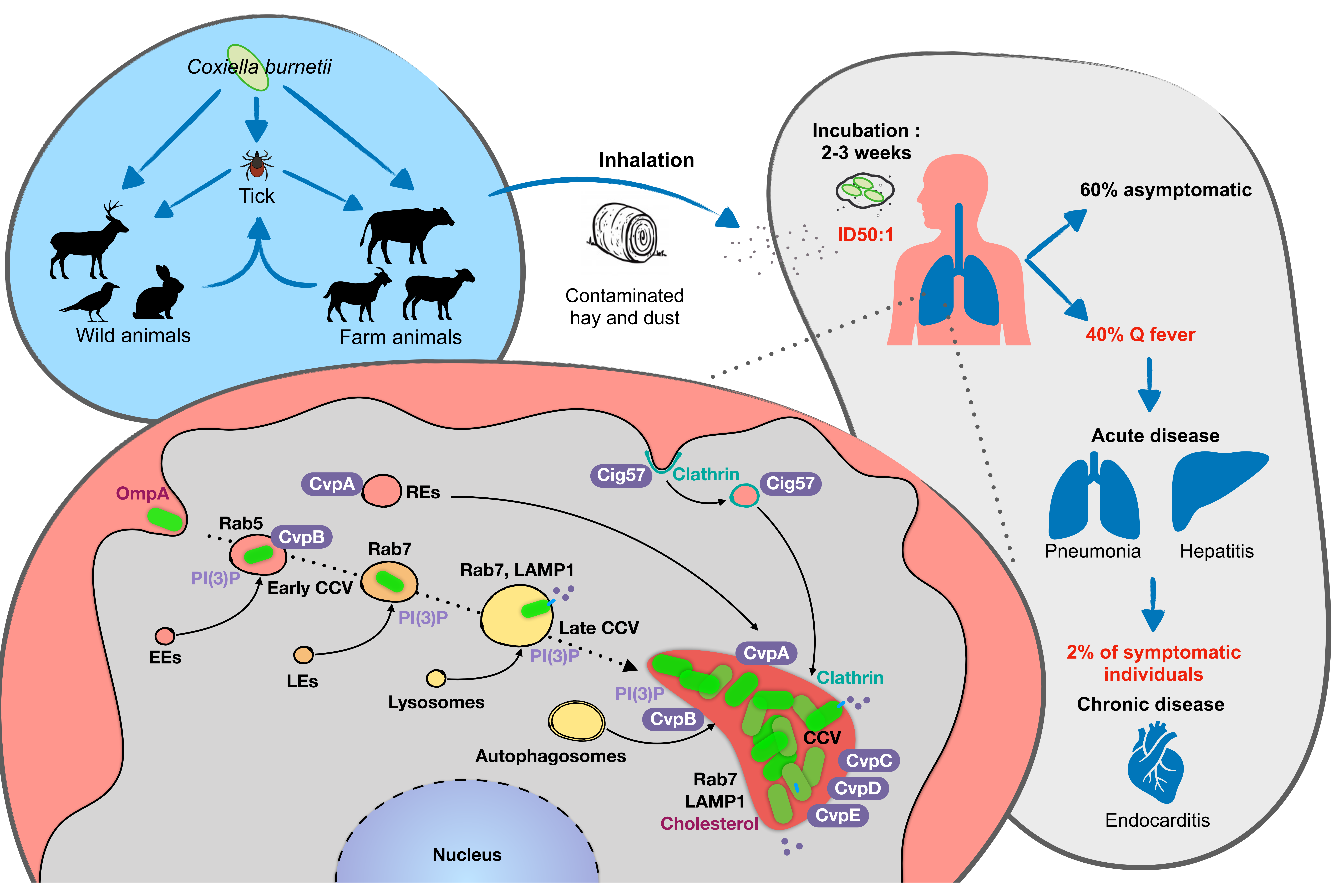




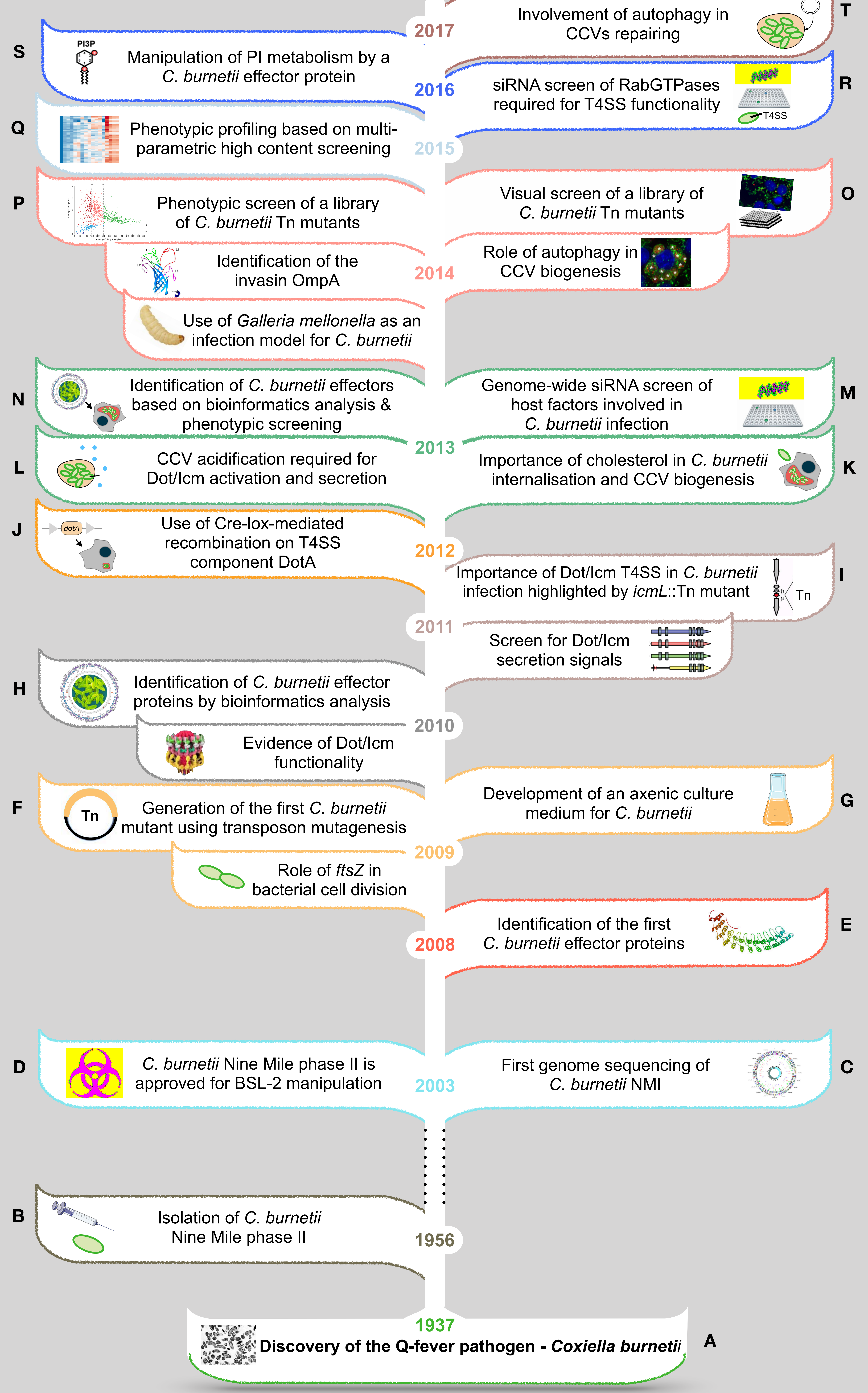

